\title{
Complement Anaphora and Dynamic Binding
}

Rodger Kibble

SOAS, University of London

\section{Complement anaphora}

The phenomenon of complement anaphora has been little studied in formal semantics (though see Kibble (1995/in press), Corblin (1996), Devlin (1997)). The most systematic investigation has been a series of psycholinguistic experiments reported by Moxey \& Sanford $(1993,1996)$, investigating what they refer to as the phenomenon of compset reference exemplified in (1a):

(1) a. Few of the MPs were at the meeting. They were too busy.

b. Few of the students were at the meeting. They soldiered on with it anyway.

Both these examples are from (Moxey, Sanford \& Barton 1990). The contrast with (1b) shows that a pronoun following a sequence few $A B$ can pick out either those $A$ 's which are $B$ (the 'reference set' or refset) or those $A$ 's which are not $B$ (the complement set or compset). Moxey \& Sanford observe that this tends to apply to quantifiers which are monotone decreasing in their right argument ${ }^{1}(M \downarrow)$ such as few, very few and not many (Moxey \& Sanford, 1996:214). Other quantifiers denoting similarly small proportions a few, only a few were found to support anaphora to the refset only (except that only a few produced some compset continuations in the presence of because). Examples (2) and (3) are attested cases which appear to involve reference to a complement set (though many readers, myself included, find (3) rather difficult to process):

(2) Not all the journalists were impressed, among them the BBC's John Simpson. (Dispatches, Channel 4)

(3) Relatively few of her films are currently available, including what was once the most celebrated, 'Never on Sunday'.

(Independent, London)

Since linguists often have difficulty is accepting the existence of this phenomenon, it is worth stressing the robustness of the experimental results. In an experiment reported in (Sanford, Moxey and Patterson 1996), subjects were presented with a series of examples of the form $Q$ of the $A$ 's were $B$. They ... and asked to provide a suitable continuation and then to indicate what they understood the pronoun to refer to (out of "refset", "compset", "A's in general", "all A's" or "other"). The results were then checked by independent judges; there were only 5 cases where judges disagreed with subjects in this and a subsequent experiment together, both experiments involving 300 subjects. The quantifiers $Q$ were chosen from a list of

(C) 1997 by Rodger Kibble Aaron Lawson (ed), SALT VII, 258-275, Ithaca, NY: Cornell University. 
"negative" (mostly $M !$ ) determiners not quite all, not all, less than half, not many and few, and a corresponding list of "positive" expressions nearly all, almost all, more than half, many and a few. The result was that $71 \%$ of responses involving "negative" quantifiers resulted in "compset" continuations, while "positive" quantifiers produced almost exclusively "refset" continuations (93\%). It is noteworthy that although subjects had the opportunity to tick "all" or "in general" hardly any chose to do so. There is an important methodological issue here, which is that data gathered from naive subjects by experimental means may turn out to contradict argumentation based on linguists' intuitions about constructed example sentences or sentence pairs.

Note that monotone increasing $(M \uparrow)$ or non-monotone quantifiers still enable the compset tobe referenced with a definite description (i.e. as a given entity):

a. Most of the fans went to the match.

b. The others watched it on TV.

So both types of quantifier partition the domain in similar ways but only compset-licensing quantifiers make both subsets available for pronominal anaphora, or place them in focus. (In this paper I follow M \& S by using the term 'focus' in a theory-neutral way to denote the set of discourse entities which are sufficiently salient that they can be picked out by a simple anaphor.) In fact the correlation with monotonicity is not absolute since it was found that the non-monotone only a few also allows compset continuations (see section 4 for discussion).

$\mathrm{M} \& \mathrm{~S}$ argue that it is not appropriate to specify the compset-licensing property in the semantics of quantifying expressions, but offer an informal explanation that quantified sentences such as few $A B$ serve to focus attention on the subset of $A$ 's which are not $B$ 's. It is after all the relative complement $A-B$ which provides evidence for the truth of few $A B$. The preferred readings are accounted for by a classification of sequences of sentences according to a rudimentary system of discourse relations WHN (what happened next - narrative continuation), and two varieties of explanation, RW (Reason Why) and RWN (Reason Why Not). M \& S observe that pronouns in RWN sentences are likely to be interpreted as 'compsets' and those in RW sentences as 'refsets'. The compset analysis is not uncontroversial, and is challenged by Francis Corblin (1996) as well as other researchers who have not committed their arguments to print. The putative examples of compset reference are claimed by these critics to be in fact generic. Corblin (op cit) argues that the purported connection between monotonicity and complement anaphora is arbitrary and ill-motivated, and that the notion of complement anaphora can be eliminated if certain independently motivated assumptions are accepted. (Similar arguments are also put forward by Percus et al 1997).

In this paper I will demonstrate that independently motivated features of a dynamic semantic account of plural anaphora allow the compset-licensing property of $M$ ! Qs to be modelled in a fairly natural way, and argue that conversational implicatures arising from interaction between Qs and context account for preferred readings. The account will be presented within the framework of Martin van den 
Berg's Generalised Dynamic Quantifier Logic (GDQL, v/d Berg 1993, 1996a, 1996b), which requires $M \downarrow$ Qs to be modelled as negations of monotone increasing $(M \uparrow)$ counterparts. The argument will stress two points in particular. Firstly, I show that for many $M \downarrow$ quantifiers $Q$ there are two alternatives available for defining the "derived" $M \uparrow$ counterparts, which I dub $Q^{d}$ and $Q^{\prime}$. These alternatives turn out to be equivalent in their truth-conditions but differ in their dynamic effects, in that $Q^{d}$ gives rise to "refset" continuations as in ( $1 \mathrm{~b}$ ) while $Q^{\prime}$ licenses complement anaphora as in (1a). Secondly, the definition for $Q^{\prime}$ relies on the relative cardinality of $A$ and $A-B$ and so it is only available in the case of proportional quantifiers; this correctly rules out compset continuations for expressions like fewer than 5 , less than $3 .^{2}$

The remainder of this paper is structured as follows. In section 2, I outline an alternative account of the data in terms of generic or $N^{\prime}$ anaphora, as proposed by Corblin (1996), and explain why I consider his account unsatisfactory. Section 3 briefly outlines the relevant features of GDQL as an account of plural anaphora and discusses the different options for representing $M !$ Qs. (This section may be skipped on a first reading without losing the gist of the argument.) Section 4 applies the results of Section 3 to the data under discussion, and includes some programmatic remarks on the analysis of expressions which do not appear to fit neatly into the framework presented in the body of the paper (such as only a few, not quite N\%).

\section{A Generic Account}

Corblin (1996) argues, within the framework of DRT (Kamp \& Reyle 1993), that the notion of "compset" can be eliminated once certain assumptions are accepted. These are: firstly that quantifiers express a normative judgment rather than simply denoting a numeric proportion, secondly that plural pronouns may be interpreted as denoting the "typical members" or the "majority" of the set defined by their linguistic antecedents, and finally that the DRT operation of "abstraction", which creates plural discourse referents, should be modified so that it selects a subset of DRS-conditions to define the referent. These assumptions are expanded below, and the ensuing claims will be criticised in section 2.5 .

\subsection{Normative readings of quantifiers}

'Vague' quantifiers like few and many are taken to denote a deviation from some contextually-specified norm or expectation. For example Few MPs came to the meeting does not simply convey the fact that some small number of MPs attended, but that the number who turned up was smaller than would normally be expected. This also appears to be the case with certain uses of numeric proportional quantifiers, as in

(5) Moins de $80 \%$ de la population a un emploi permanent. 'Less than $80 \%$ of the population have a permanent job'. 
According to Corblin, this seems to imply that one would normally expect more than $80 \%$ to be employed.

\subsection{Generic interpretation of plural pronouns}

Corblin assumes that predications of plural pronouns with antecedents of indeterminate cardinality effectively include a hidden generic operator, so that in example (6) They doesn't mean all students without exception but something more like most students, students in general:

(6) I've taught thousands of students and I can assure you they were lazy. ... Well, most of them anyway.

Percus et al (1996) claim that this also applies to cases of definite reference:

Only 20 of the 30 cows gave milk. The herd's unproductivity was surprising.

The argument here is that even though only 10 of the 20 cows in the herd did not produce milk, the herd as a whole can be termed 'unproductive' since its productivity was less than desired or normal.

\section{3. 'Partial' DRS-abstraction}

Kamp \& Reyle (1993:309ff) propose a rule of Abstraction which essentially creates plural discourse referents licensed by quantified NPs. This is motivated by examples like (8):

(8) Susan has found every book which Bill needs. They are on his desk.

The process is illustrated by the schematic DRS exhibited in (9), where $\phi$ is to be read as " $x$ is a book which Bill needs", $\psi$ as "Susan has found $x$ " and $\chi$ as " $x$ is on his [Bill's] desk".

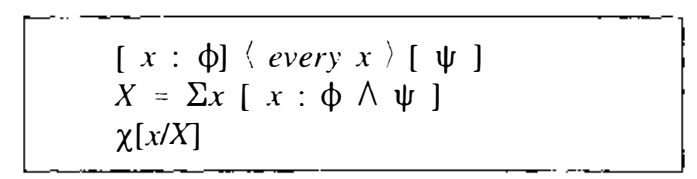

Thus the plural referent $X$ is set up by 'abstracting' over the set defined by the conjunction of restriction and nuclear scope of the quantifier. The abstraction operator $\Sigma$ creates a plural referent $X$ by "summing" the values of $x$ which satisfy $\phi \wedge \psi$. So in this example They doesn't simply mean books which Bill needs but books which Bill needs and Susan has found (though these sets turn out to be co-extensive). Clearly this is not the only possibility, and Corblin proposes a notion of 'partial abstraction' which picks out a subset of the DRS conditions to define the 
discourse referent. This is motivated with reference to Kamp \& Reyle's example (10), where the pronoun they appears not to refer to the set of 'women from the village who came to the feminist rally' but rather 'women from the village in general' or perhaps 'women in general'.

(10) Few women from this village came to the feminist rally. No wonder. They don't like political rallies very much.

$[x: \phi]\langle$ Quant $x\rangle[\Psi]$
$X_{1} \cdot \Sigma x[x: \phi \wedge \psi]$
$X_{2}=\Sigma x[x: \phi]$

This account allows a pronoun to refer to the 'maxset' in Corblin's terminology, which is all (or a majority) of the restrictor set. (Note that this approach probably requires some structure to be imposed on the DRS-conditions, to prevent the creation of discourse referents such as for example $X_{3}=\Sigma x[x: \psi]$, or "those entities which came to the feminist rally" in example (10).)

\subsection{Eliminating COMPSET}

Corblin's claim is that the combination of the above innovations allows the Moxey \& Sanford data to be accounted for without appealing to the notion of 'complement set'. The quantifiers classified by $\mathrm{M} \& \mathrm{~S}$ as 'compset licensing' are those which imply that the 'refset' is smaller than expected on the grounds of some contextual norm. The effect is to 'defocalise' the refset $A \cap B$ and trigger a process of 'partial abstraction' resulting in a discourse referent $X$ which picks out the 'maxset' $A$, equivalent to $N^{\prime}$ anaphora. A subsequent plural pronoun is understood to refer to $A$ in general, or to the 'typical' members of $A$. Since the 'refset' $A \cap B$ has been designated as deviating from the norm then the actual set of 'typical $A$ 's' which is selected for pronoun resolution effectively coincides with the 'compset' $A-B$.

To give an example, the claim is that (12) below is understood as most MPs or MPs in general were at the pub or with their secretaries.

(12) Few MPs came to the meeting. They were at the pub or with their secretaries.

The fact that this is effectively the same as 'the MPs who did not come to the meeting' is supposed to explain why experimental subjects thought they were referring to this set rather than to the 'maxset'. Percus et al (1997) also claim that apparent cases of complement anaphora are in fact generic cases where the subset verifying a predication is indistinguishable from the complement set.

\subsection{Critique of the generic account}


A rather straightforward objection to the "generic" explanation is that one should expect the same readings to be available with a definite MP substituted for the pronoun, as in (12'):

(12') Few MPs came to the meeting. \#The MPs were at the pub or with their secretaries.

However this is clearly unacceptable, and requires the insertion of some such expression as mostly or in general. ${ }^{3}$ There are at least further two points on which the generic argument as presented by Corblin is open to criticism (for further discussion see Moxey \& Sanford 1996b):

\subsubsection{Compset can be a minority of the domain}

Corblin argues that his argument is consistent with $M$ \& S's data concerning numeric proportional quantifiers: with e.g less than $20 \%$ there is a strong preference for compset continuations but hardly any with less than $80 \%$. This is to be expected if it is in fact the 'majority' set which is picked out and not in fact the complement set. However, $M$ \& S adduce evidence which appears to contradict this, involving the quantifier not quite all:

(13) a. Not quite all of the MPs were at the meeting.

b. They stayed at home instead.

In this case it is the "small, exceptional group" who stayed at home (M \& S, 1993:64) and so it can hardly be argued that the "majority set" is being conflated with the "complement set" in this instance. Striking confirmation of this line of argument is provided by a more recent study contrasting not quite $N \%$ with nearly $N \%$ where $N$ ranged from 10 to 90 (Sanford, Moxey \& Patterson 1996). (This study will be more fully discussed in section 4 ).

\subsection{2. "Downward normative" quantifiers are not always compset-licensing.}

One can find or construct examples where a quantifier expresses 'smallness' compared to some norm or expectation but does not give rise to a complement reading. The following two examples are both attested, (14) from the COBUILD corpus and (15) from the Brown corpus as maintained by the Linguistic Data Consortium.

(14) While a few became richer, many did not.

(Berry 1997:133)

Here the connective 'while' appears to signal that it is the people who became rich who constitute the abnormal group. In fact this sentence could easily be paraphrased as 'Only a few got richer, but many did not'. 
(15) Even with the increase in funds for the next fiscal year, Georgia will be spending only around $\$ 3.15$ per day per patient. The national average is more than $\$ 4$ and that figure is considered by experts in the mental health field to be too low. Kansas, regarded as tops in the nation in its treatment of the mentally ill, spends $\$ 9$ per day per patient . Georgia has made some reforms, true. The intensive treatment program is working well. But in so many other areas we still are dragging.

(Brown Corpus, position 109998)

In example (15) the highlighted phrase some reforms implies that the number of reforms made is less than the speaker considers desirable. But it is clearly impossible for a subsequent pronoun They to be understood as referring to 'the reforms which still need to be made', for instance. From these two examples it appears that the implicature of relative 'smallness' is not carried by the quantifier alone but by the combination of quantifier and context; in these cases some and a few are explicitly contrasted with many. (In Gricean terms, the implicature is conversational not conventional.) On the basis of these examples I would argue (contra $\mathrm{M} \& \mathrm{~S}$ as well as Corblin) that the compset-licensing property is separate from normative implicatures carried by uses of quantifying expressions. In the remainder of this paper I will give an alternative account where the anaphoric potential of quantified NPs is more closely related to fundamental semantic properties.

\section{Dynamic Semantics for Plural Anaphora}

This section briefly introduces Martin van den Berg's GDQL (Berg 1993, 1996) which is a systematic attempt to incorporate Generalised Quantifier (GQ) theory into a dynamic plural logic. The use of GQs to represent the semantics of noun phrases has been fairly widely accepted since the work of Montague (1973) and Barwise and Cooper (1981). However, van den Berg makes it clear that GQs on their own do not capture all the required properties of NPs in multi-sentential text, but need to be supplemented with a notion of maximality and some mechanism for introducing and keeping track of discourse referents. Under standard linguistic accounts of GQs, a sentence of the form Det $N V P$ is interpreted as asserting that the set denoted by $V P$ is a member of the set of sets denoted by Det $N$ : for instance Many men sleep is true if the set of sleepers is one of the set of sets which include many men. But this doesn't automatically make available any semantic object which could serve as the interpretation of a subsequent plural pronoun They; neither the set of sleepers, nor an arbitrary set including many men is an appropriate candidate. Previous accounts of intersentential anaphora in the "E-Type" tradition have supplemented a truthconditional semantics for quantified sentences with some inferential technique for constructing (hypothesising) a resolvent for pronouns at the point where they are encountered, by recovering the descriptive content of previous NPs (see for example Neale 1990, van der Does 1993 for recent accounts). The strategy followed by 
dynamic accounts (of which GDQL is a representative) is to set up a more complex analysis of quantified NPs, such that the relation denoted by a generalised quantifier is tested for a suitably restricted domain, and sets which satisfy the test will be appropriate candidates for subsequent anaphora resolution.

A maximality requirement is imposed for $M \uparrow$ quantifiers; this is partially motivated on the grounds that v/d Berg provides coverage of collective as well as distributive readings of quantifiers, so that for example Three men lifted a piano will not come out true on the collective reading if it was a group of four men who lifted the piano. On the other hand, in the case where several groups of people lifted a piano we want to be able to pick out a particular group by saying e.g. Four men lifted a piano. They...; and we don't want this sentence to turn out false if there happens to be some other group of piano-lifters numbering more or less than four. So there need not be a unique maximal set defined by the quantified sentence but rather a number of local maxima which provide alternative resolvents for subsequent pronouns. ${ }^{4}$ In both the static and dynamic cases, $M !$ quantifiers are defined as the negation of $M \uparrow$ quantifiers on the grounds that $M !$ quantifiers defined directly do not define a unique maximal set corresponding to the nuclear scope and that this may result in spurious verification of a quantified sentence. This is discussed further in section 3.2 .

The system interprets formulas as defining a relation on pairs of 'plural contexts' $(G, H)$ each consisting of sets of variable assignments $g$; apart from this the principal innovation is that the variables in the domain of $g$ range over plural objects (sets) rather than individuals. This allows for collective plural readings only. To get distributive readings and support singular pronouns v/d Berg introduces extra operators $\Delta$, which 'breaks down' plural objects to allow dependencies to be stated, and sing, which allows individuals to be treated as singleton sets. The collective reading is primary and requires maximal sets to resolve pronouns and the maximality condition is automatically inherited in the distributive and singular cases.

Thus v/d Berg's basic definition of dynamic generalised quantifiers looks like this Berg (1993:134), where $M$ is a maximalisation function, $\epsilon_{x}$ is the dynamic existential quantifier and $Q\left(x^{\prime}, x\right)$ is a corresponding static quantifier.

\section{Definition 1: Dynamic Quantification}

$$
Q,(\phi, \Psi):=\epsilon_{a} \wedge \epsilon_{x^{\prime}} \wedge\left(M x^{\prime}\left(\phi\left[, / x^{\prime}\right]\right) \wedge M x\left(x=x^{\prime} \wedge \psi\right) \wedge Q\left(x^{\prime}, x\right)\right.
$$

Roughly speaking, a dynamic quantifier $Q_{x}(\phi, \psi)$ comes out true in a context $G$ yielding an output context $H$ if there is some $h \in H$ such that $h(x)$ is a maximal set satisfying $\psi$ and $h(x)$ is a subset of some $h\left(x^{\prime}\right)$ which is a maximal set satisying $\phi$. The operator $M$ ensures that the output set is maximal; i.e. for any set $g(x), g \in G$ which satisfies $\phi \wedge \psi$, there is no $y$ s.t. $g(x) \subsetneq g(y)$ and $g(y)$ satisfies $\phi \wedge \psi$. Since $H$ becomes the input context for evaluating subsequent discourse, all 
qualifying values of $x, x^{\prime}$ are passed on as potential resolvents of pronouns.

\subsection{Maximality vs uniqueness}

As was just noted, van den Berg's theory takes collective plural quantification as the basic case and introduces additional operators to deal with distributive and singular quantifiers. This means that the distributive definition 'inherits' maximality from the collective one where it is obviously required. But at first sight it is questionable whether this is right for distributive readings. Compare examples (16) and (17):

(16) John met some girls yesterday. They invited him to dinner.

(17) John owns some sheep. Harry vaccinated them.

In (16) the girls who invite John to dinner are not necessarily all the girls he met the previous day, though they are all the members of a particular group of girls. In (17) on the other hand the pronoun them is understood to refer to all the sheep John owns. Both these examples involve the determiner some so we can't appeal to a distinction between indefinites and quantified NPs. In fact GDQL caters for both these cases, although the theory as presented in (van den Berg, 1996) doesn't specify when one or the other reading can be predicted. On the assumption that they picks up a maximal discourse referent, which is itself a plural object, then (16) concerns the entirety of some group of girls who John has met; it doesn't have to mean all the girls he met on that day since they may not constitute a group which he encountered all at once. On the other hand (17) concerns a maximal group of sheep which John owns, which coincides exactly with the set of all sheep which he owns. This is an informal way of characterising the difference; closer consideration of the examples suggests two possible distinct or overlapping explanations:

1. The difference in anaphoric potential may be accounted for by the predicate rather than the type of quantifier: in (17) the sheep have the individual-level property of 'being owned by John' which defines a unique maximal set, as opposed to the stage-level property of 'being met by John on a particular day' which does not.

2. On the other hand the differences in tense and aspect between the two examples may be the key factor: (16), in the past tense refers to an event while the first sentence of (17) in the present tense describes a state. The fact that (16) allows for multiple maximal sets may be accounted for by scope interaction between the quantifier and an event(uality) argument.

\subsection{Monotone decreasing quantifiers}

The following example (v/d Berg, 1996) shows that Definition 1 is not restrictive enough for $M$ ! quantifiers, which have to inspect all values of $H(x)$ rather than checking that at least one satisfies the requirements.

At most three women gather in the square. 
According to Definition 1 this would be satisfied if some group of three women or fewer gathers in the square, even if there are larger groups around. The solution proposed by v/d Berg is to define $M !$ quantifiers as negations of their $M \uparrow$ 'duals', as follows:

\section{Definition 2: Static $M !$ Quantification} (adapted from v/d Berg (1996:92)

$\neg \exists x, x^{\prime}\left(M x^{\prime}\left(\phi\left[x / x^{\prime}\right]\right) \wedge M x\left(x \subseteq x^{\prime} \wedge \psi\right) \wedge Q^{d}\left(x^{\prime}, x\right)\right.$ where $Q^{d}$ is the 'dual' of $Q$.

We can illustrate this by considering the quantifier few (as defined by Lappin 1993):

\section{Vague quantification: Monotonic $f e w$}

$$
B \in \| \text { few }(A, C) \| \text { iff }|A \cap B|<|A \cap C|
$$

where $A$ is the $N^{\prime}$ set, $B$ the $V P$ set, $C$ a contextually determined comparison set.

\section{Static $f e w^{d}$}

$$
B \in \| \text { few }(A, C) \| \text { iff }|A \cap B| \geq|A \cap C|
$$

In fact this definition of the 'dual' of few is identical to Lappin's (1993) definition 5b for many.

Definition 2 works well enough for static quantification: what it says essentially is that there are no appropriate values for $x, x^{\prime}$ which make the conjunction true. However, we need to be a little careful in adapting this to dynamic quantification. As Berg (1993:139) acknowledges, some $M \downarrow$ quantifiers result in a "strange mix of values [for] $x$ in different states". Suppose for example we simply reformulate Definition 2 with dynamic existential quantification, giving Attempt 1 :

\section{Attempt 1: Dynamic $M \downarrow$ Quantification}

$Q_{x}(\phi, \psi):=$

$\neg\left(\epsilon_{x} \wedge \epsilon_{x^{\prime}} \wedge\left(M x^{\prime}\left(\phi\left[x / x^{\prime}\right]\right) \wedge M x\left(x \subseteq x^{\prime} \wedge \psi\right) \wedge Q^{d}\left(x^{\prime}, x\right)\right)\right.$

The definition of negation assumed in these definitions is dynamic, i.e. the dynamic existential quantifiers $\epsilon_{x}, \epsilon_{x}$ set up discourse referents corresponding to $\phi$ and $\phi \wedge \psi$ respectively, which are accessible outside the scope of negation. So while Attempt 1 is truth-conditionally equivalent to Definition 2, the dynamic effect is that $x, x^{\prime}$ pick up any values which make the conjunction as a whole false. ${ }^{5}$ The danger is that irrelevant values may be passed on. For instance, suppose there is a value of 
$x^{\prime}$ such that $M x^{\prime}\left(\phi\left[x / x^{\prime}\right]\right)$ is false $-x^{\prime}$ is not a maximal set of $\phi$-ers, but the other conjuncts are true. This would have the effect in example (1a) that the pronoun They would pick up neither all the MPs who went to the meeting, nor all those who didn't, but some group of MPs who went to the meeting and make up proportionately 'many' of the arbitrary group of MPs picked out by $x^{\prime}$.

Given that $M \downarrow$ quantifiers are interpreted 'indirectly' via translation into a more complex formula, we may point out that the definition above is not the only possibility. Different possibilities exist for the precise definition of the 'derived' quantifier and the placement of the negation operator. We will make use of derived quantifiers discussed by (Zwarts 1996), the 'complement' $-Q$ and the 'contradual' $Q$ - Suppose (standardly) a quantifier $Q$ denotes a set of subsets of the domain of discourse $\mathrm{U}$. Then the derived quantifers $-Q$ and $Q$ - are defined as follows:

1. $-Q$ is the set of subsets $X$ of $U$ s.t. $X \notin Q$. For example, if $Q$ is less than $20 \%$ of $A$ 's then $-Q$ is $20 \%$ or more of $A^{\prime} s$. For $Q=$ less than $X,-Q$ is $X$ or more; for $Q=$ not all, $-Q$ is all; for $Q=$ neither $N,-Q$ is either (of) $N$

2. $Q$ - is the set of subsets $X$ of $U$ s.t. $-X \in Q$. For example, if $Q$ is less than $20 \%$ of $A$ 's then $-Q$ is $80 \%$ or more of $A$ 's. For $Q=$ not every, $Q$ - is some; for $Q=$ neither $N, Q$ - is both $N$.

First of all, it can be seen that $-Q$ corresponds to $Q^{d}$ which we have used in Definition 2 and Attempt 1 above. So we shall proceed by first setting out a workable definition using $-Q$ and then see what happens if we take $Q$ - as the basis. It is questionable whether the negation operator needs to apply to the whole conjunction or whether only individual conjuncts or sub-formulas should be negated. In fact we don't want to negate the formulas which set up the discourse referents since it is indisputable that anaphoric reference is possible both to the domain set defined by $\phi$ and to the 'intersection' set defined by $\phi \wedge \psi$ (cf example lb). The negation of a conjunction allows one or more of its conjuncts to be true; in practice we might initially expect to find that $M \downarrow$ quantification reduces to the special case below. The reason for this being that for anaphora resolution we need $x^{\prime}, x$ to be resolved to maximal witnesses for $\phi, \phi \wedge \psi$ and only the 'static' quantificational test should be negated.

\section{Attempt 2: Dynamic $M !$ quantifiers: External Negation}

$Q,(\phi . \psi):=$

$$
\epsilon_{x} \wedge \epsilon_{x} \wedge\left(M x^{\prime}\left(\phi\left\{x / x^{\prime}\right\}\right) \wedge M x\left\{x \leq x^{\prime} \wedge \psi\right) \wedge \neg Q^{d}\left(x^{\prime}, x\right)\right.
$$

In fact this isn't sufficient either: although it seems to get the dynamic effects right it is not restrictive enough, since it requires some witness set $x$ of which $\neg Q^{d}\left(x^{\prime}, x\right)$ holds, rather than ensuring that $Q^{d}\left(x^{\prime}, x\right)$ holds for $n o$ witness set. It looks as if we need to combine the static definition which checks all the maximal witness sets with a separate dynamic component which makes the surviving sets available for anaphora resolution:

Definition 3 (Dynamic $M \downarrow$ Quantification: a merged definition) 
$\neg \exists x, x^{\prime}\left(M x^{\prime}\left(\phi\left[x / x^{\prime}\right]\right) \wedge M x\left(x \subseteq x^{\prime} \wedge \psi\right) \wedge Q^{d}\left(x^{\prime}, x\right)\right)$

$\wedge \epsilon_{x} \wedge \epsilon_{x^{\prime}} \wedge\left(M x^{\prime}\left(\phi\left[x / x^{\prime}\right]\right) \wedge M x\left(x \subseteq x^{\prime} \wedge \psi\right)\right)$

This proto-definition is inelegant and contains some repetition and can hopefully be regarded as provisional. However it appears to be 'safe' as far as the dynamic effects are concerned, without weird unintended side-effects. The definition has a 'static' part prefixed by the standard existential quantifier $\exists$ which says that the dual $Q^{d}$ does not hold over the sets defined by $\phi$ and $\phi \wedge \psi$, and a 'dynamic' part introduced by the dynamic quantifier $\epsilon$ which sets up the 'discourse referents' $x$ ' which verifies $\phi$ and $x$ which verifies $\phi \wedge \psi$. So this definition supports 'refset' continuations in M \& S's terminology; for example in (1b) the pronoun They picks out the maximal group of few students who were at the meeting.

We should point out however that in certain cases the placement of the negation operator is in a sense arbitrary as far as the 'static' truth-conditional effects are concerned; for instance we may consider Definitions 4 and 5 below, where $\psi$ is negated and $Q^{\prime}$ corresponds to Zwarts' 'contradual' $Q$-:

\section{Definition 4 (Static $M !$ Quantification: Internal Negation)}

$\neg \exists x, x^{\prime}\left(M x^{\prime}\left(\phi\left[x / x^{\prime}\right]\right) \wedge M x\left(x \subseteq x^{\prime} \wedge \neg \psi\right) \wedge Q^{\prime}\left(x^{\prime}, x\right)\right.$

We illustrate the interpretation of $Q^{\prime}$ using the example of few:

\section{Static few'}

$$
B \in \| \text { few }(A, C) \| \text { iff }|A-B|<|A \cap C|
$$

NB: unlike $f e w^{\prime \prime}$ this definition relies on the cardinality of $A-B$. For any sentence $\left[Q_{x} \phi\right] \psi$, where $Q$ is $M !$, we obtain some $\left[Q_{x}^{\prime} \phi\right] \neg \psi$, with Q' $M^{\dagger}$. E.g. not all men ran $\rightarrow$ some men did not run; few men ran $\rightarrow$ many men did not run (informally; in fact $f e w^{\prime}$ as defined above may not have an exact NL equivalent.) less than $20 \%$ of the men ran $\rightarrow$ more than $80 \%$ of the men did not run. In each case a witness set for $\left[Q_{x}^{\prime} \phi\right]$ verifying $\left[Q_{x}^{\prime} \phi\right] \neg \psi$ also verifies $\left[Q_{x} \phi\right] \psi$. In contrast to Definition 3 , a dynamic version is obtained fairly straightforwardly by substituting the dynamic existential quantifier $\epsilon$ for the standard quantifier $\exists$.

\section{Definition 5 (Dynamic $M !$ quantifiers: Internal Negation)}

$Q_{x}(\phi, \psi):=$

$$
\epsilon_{x} \wedge \epsilon_{x^{\prime}} \wedge\left(M x^{\prime}\left(\phi\left[x / x^{\prime}\right]\right) \wedge M x\left(x \subseteq x^{\prime} \wedge \neg \psi\right) \wedge Q^{\prime}\left(x^{\prime}, x\right)\right.
$$

\section{Remarks on Definitions 4 and 5}

Definition 5 appears to be truth-conditionally adequate by the above equivalences. This does give rise to different dynamic effects from Definition 3 however and the 
next question is to establish whether or not these are appropriate. In fact what happens here is that the values of $x^{\prime}, x$ passed on in the output are the 'maxset' $\lambda x^{\prime} \phi\left[x / x^{\prime}\right]$ and the complement set $\lambda x(\phi \wedge \neg \psi)$ respectively. So in example (1a) the result of applying this definition is that They may refer to those MPs who were not at the meeting.

\section{Dynamics of $M \downarrow$ quantifiers}

Here we repeat the different interpretations of $(1 \mathrm{a} / \mathrm{b})$ (repeated as $(19 \mathrm{a} / \mathrm{b}))$

afforded by the definitions given above.

(19) a. [Few of the MPs] $]^{x}$ were at the meeting. They $y_{x / x}$ were too busy.

b. [Few of the students] $]^{y}$ were at the meeting. They $y_{y / y^{\prime}}$ soldiered on with it anyway.

\subsection{Alternative readings}

The first sentence of (19a) is paraphrased according to Definition 3 as (roughly) "the set of MPs who came to the meeting $(\lambda x(\phi \wedge \psi))$ was not a set of many of the MPs". Following Definition 5 the paraphrase would be "the set of MPs who did not come to the meeting $(\lambda x(\phi \wedge \neg \psi)$ ) was a set of many of the MPs". If we assume that many is a dual of few then these paraphrases are equivalent as far as truth-conditions are concerned but differ in their anaphoric potential as.is spelled out below.

$G \|[\text { Few of the MPs }]^{\mathrm{x}}$ were at the meeting $\| H$

by Def 3, 5 They ${ }^{x^{*}}=H\left(x^{\prime}\right)=$ MPs in general

by Def 5 They ${ }^{\mathrm{x}}=H(x)=$ MPs who were not at the meeting

$G \|[$ Few of the students] $y$ were at the meeting $\| H$

by Def 3, 5 They ${ }^{{ }^{*}}=H\left(x^{\prime}\right)=$ students in general

by Def 3 They ${ }^{y}=H(y)=$ students who were at the meeting

So it appears that Def 5 gives the right reading for (19a) and Def 3 gives the appropriate reading for (19b).

At this point we could sum up as follows: we have demonstrated that there is no obviously unique translation procedure for sentences including $M$. quantifiers, and the two proposed definitions seem to give appropriate readings in differing cases. So we could simply treat $M !$ quantifiers as ambiguous between the two readings, where the ambiguity is not evident in the truth-conditions but only in the dynamic effects.

However, we have observed that Def 5, unlike Def 3 , depends on the cardinality of $A$ and is not applicable in cases like (20) where the cardinality is not known: 
(20) Few/less than 5 carol singers came to my door last night. It must have been too cold for them.

where them can only mean 'carol singers in general' rather than 'carol singers who didn't come to my door'.

Following Def 3 this sentence is verified by counting the carol singers and checking that the total come to less than would normally be expected. But if Def 5 is selected then verification requires identifying all the carol singers (in whatever domain) and checking that their cardinality corresponds to the complement of 'few' namely something like 'at least all except few of them'. This clearly relies crucially on the cardinality of the domain $A$ which is only vaguely specified and may not be precisely identifiable: in fact there may be no carol singers at all.

To summarise, Def 5 is not generally applicable since there is no general, context-independent procedure for verifying the complement of "cardinal" quantifiers like few $N$, less than $N$ where the cardinality of the domain may not be known, and so the phenomenon of complement anaphora is accordingly limted.

\subsection{Monotonicity reconsidered}

There is experimental and anecdotal evidence that downward monotonicity is not in fact a necessary or even a sufficient condition for complement anaphora; these are illustrated in examples $(21-24)$ :

(21) Only a few of the fans went to the football match, because they ...

(Moxey and Sanford 1993)

(22) Not quite all of the football fans went to the game.

They were drunk and lay unconscious on the pavement.

(Sanford, Moxey and Patterson, 1996.)

(23) Neither student submitted a paper. They couldn't be bothered.

(Shalom Lappin, p.c.)

(24) At most half of the MP's came to the meeting. They ...

(Manfred Pinkal, at SALT VII workshop)

\subsubsection{Non-monotone quantifiers}

As observed in the introduction the quantifier only a few licenses compset reference, though only in the presence of because; while intuitively it seems to be non-monotone:

(25) a. Only a few of the guests wore carnations

b. Only a few of the guests wore white carnations

gives rise to no entailment in either direction. This suggests that the compset licensing property is not determined by monotonicity but there is some more fundamental factor of which proportional $M !$ quantifiers form a special case. This 
would also apply to expressions of the form not quite all, not quite N\% which are shown by (Sanford, Moxey and Patterson 1996) to produce compset continuations, in contrast to nearly $N \%$ which is virtually indistinguishable from not quite $N \%$ as far as truth-conditions are concerned.

What only a few has in common with the $M \downarrow$ quantifiers is that (in the framework adopted here) it has to be interpreted indirectly via some translation into a complex expression in the quantifier language, and that different possibilities exist which are truth-conditionally equivalent, including $(26 a, b)$ :

a. [at least a few and no more than a few] A's are B
b. [all except a few] A's are not B

As in the $M \downarrow$ cases these alternatives differ primarily in their dynamic effects: option (a) sets up as a potential antecedent the A's which are B, and option (b) passes on the A's which are not B. A tentative solution, which I do not develop here, is that nonmonotone quantifiers in general should be analysed as conjunctions of $M \downarrow$ and $M \uparrow$ quantifiers, with variation as to which component contributes the "dynamic" effect. Of course this will require some care to prevent over-generation in the case of those non-monotone Qs which do not license complement anaphora, such as exactly $N$. Formal analysis of the difference between the (non-monotonic) nearly X\% and not quite $X \%$ cases may show that it is a pragmatic factor of negativity which is critical and that the $M !$ quantifiers form a special case of the compset-licensers. Another subject for further research is the precise effect of discourse connectives such as because, while, but etc.

\subsubsection{Troublesome $M !$ quantifiers}

Shalom Lappin (p.c.) argues that in example (23) the pronoun They following the $M$ ! Neither student can only have a "refset" interpretation, where he takes the "refset" to be the set of $N$ 's which satisfy the VP (a verifying "witness set"). I think this partly comes down to a difference in terminology; in $M \& S$ 's terms the "refset" for a sentence $Q A B$ is the intersection of $A$ and $B$ (regardless of the value of $Q$ ), while in Lappin's example the "two students" denoted by They constitute the "maxset" $A$ which happens to be co-extensive with the compset $A-B$. This is an unresolved dispute, but I think the root of the problem is that GQ theory on its own has nothing to say about what potential antecedents are made available by a quantifying expression and needs to be supplemented either by an "inferential" account in the Etype tradition or by a dynamic account such as GDQL where the semantics determines in advance what referents are available for pronoun resolution. In the system I have proposed, (23) is uninterpretable under Definition 3 (since there is no non-null intersection set to satisfy the dynamic component of the definition) but is, I claim, correctly interpreted under Definition 5 as (roughly) "Both of the two students did not submit a paper. Those two students couldn't be bothered".

Finally, the $M \downarrow$ determiner at most $P$ ( $P$ a fraction or percentage) appears not to license compset continuations, in contrast to less/fewer than $P$ : 
(27) a. At most a quarter of the students came to the inaugural lecture. ?\#They were all at the pub.

b. Less than a quarter of the students came to the inaugural lecture. ?They were all at the pub.

At this point I do not propose an explanation for this, but there are two possible lines of enquiry. Firstly, I have already mentioned that experimental evidence shows the phenomenon of complement anaphora to be surprisingly widespread, and it is unwise to uncritically accept constructed examples as counter-evidence; it would be interesting to include at most $P$, no more than $P$ etc in experiments of the kind conducted by $M \& S$ to determine whether naive subjects do after all produce compset continuations in an appropriate context. Secondly, if it turns out that these continuations are completely ruled out then further investigation is needed into pragmatic factors which in most cases favour either Definition 3 or Definition 5 readings for $M \downarrow$ quantifiers, and in some cases may rule out the Definition 5 reading altogether.

\section{Conclusion}

This paper comprises two converging strands of argumentation: firstly we reviewed the empirical evidence for the phenomenon of complement anaphora and argued against the claims that only $N$ 'or generic anaphora is involved, and subsequently we demonstrated that this phenomenon can be modelled fairly naturally in the framework of GDQL. In that framework dynamic $M !$ quantifiers are not given a direct definition but are defined as negations of $M \uparrow$ quantifiers; however there is no obviously unique method for defining the negations, and different choices lead to different dynamic effects which correspond to $M$ \& S's 'refset' and 'compset' readings. However, both alternatives allow for a 'generic' resolution of plural pronouns via the $x^{\prime}$ variable in addition to the quantifier reading. A task that remains is to investigate (pragmatic/inferential) factors which cause a particular reading to be favoured. This work has also raised more general methodological issues, which are discussed further in the final chapter of Kibble 1997. The data discussed here originally arose from empirical psychological research rather than in response to any linguistic theory, and indeed linguistic researchers tend to be surprised by the extent of the evidence for the existence of complement anaphora. There is evidently scope for further interaction between theoretical linguists and psychological researchers, both to provide theoreticians with more objective and reliable sources of data and to provide psycholinguists with the formal tools to frame more prccise hypotheses.

\section{Endnotes}

* Thanks to Martin van den Berg, Naomi Devlin, Howard Gregory, Ruth Kempson, Shalom Lappin, Lutz Marten and Anna Pettiward for helpful feedback on earlier 
presentations and drafts of this material, as well as the SALT reviewers and participants in the SALT VII workshop. This work was carried out while I was employed on the project "A Labelled Deductive System for Natural Language Understanding", funded by the UK Engineering and Physical Sciences Research Council under grant reference GR/K67397.

1. A quantifier $Q$ is said to be monotone decreasing if for any sets $A, B, C: Q(A, B)$ implies $Q(A, B \cap C)$; for example few men ran implies few men ran quickly.

2. Counter-examples involving less than $N$ have been proposed in the past by Remko Scha and Craige Roberts, among others.

3. This observation is due to Orin Percus (p.c.). It is interesting to note that the generic interpretation appears to be readily available with possessive pronouns/NPs:

(i) Few MPs came to the meeting.

(ii) Their/The MPs' discourtesy annoyed the committee members.

However, this may be restricted to "individual-level" predicates which favour a generic reading; compare the following examples with a "stage-level" predicate substituted:

(iii) Few MPs arrived on time.

(iv) \# Their/The MPs' lateness annoyed the committee members.

4. A consequence of this analysis is that there may be no unique "refset" but rather a number of "witness sets" which are available for anaphora resolution (cf Devlin 1997).

5. Martin van den Berg has argued (p.c.) that his definition of dynamic negation (van den Berg 1996b) severely restricts anaphoric potentials, so that it is not the case that Attempt 1 would be satisfied by arbitrary values for $x$. In fact the analysis I propose avoids the use of dynamic negation and so this issue does not arise.

\section{References}

Barwise, J and R Cooper, 1981, Generalized Quantifiers and Natural Language, Linguistics and Philosophy 4, 159 - 219

Berg, M van den, 1993. A Direct definition of dynamic generalised quantifiers, in Dekker, P and M. Stokhof (eds), Proceedings of the 9th Amsterdam Colloquium, pp. 121-140, ILLC, Amsterdam

Berg, M van den, 1996a, Dynamic generalised quantifiers, in J. van der Does and J van Eijck, (eds), Quantifiers, Logic and Language, pp 63 - 94, CSLI, Stanford.

Berg, M van den, 1996b, The Internal Structure of Discourse, PhD thesis, Universiteit von Amsterdam.

Berry, R, 1997, Determiners \& Quantifiers, COBUILD English Guides, HarperCollins, London.

Corblin, F, 1996, Quantification et anaphore discursive: la référence aux complémentaires, Langages 123, pp 51 - 74, Larousse, Paris.

Devilin, N, 1997, Pronominal Anaphora Resolution and the Quantified Phrase, Master's thesis, University of California at Santa Cruz. 
Does, J van der, 1993, Formalising E-type anaphora, in P. Dekker and M. Stokhof (eds), Proceedings of the 9th Amsterdam Colloquium, 229-248, ILLC, Amsterdam.

Kamp, H \& U Reyle, 1993, From Discourse to Logic, Kluwer, Dordrecht.

Kibble, R, 1995/in press, Modal Subordination and Complement Anaphora, to appear in J. Ginzburg, Z Khasidashvili, C Vogel, J-J Lévy and E Vallduvì, (eds), Selected papers from the Tbilisi Symposium on Logic, Language and Computation, CSLI, Stanford.

Kibble, R, 1997, Anaphora Resolution in Dynamic Semantics, PhD thesis, Centre for Cognitive Science, University of Edinburgh.

Lappin, S, 1993, 'Many' as a two-place determiner function, in Working Papers in Linguistics and Phonetics 3, pp 337 - 358, SOAS, University of London.

Montague, R, 1973, The Proper Treatment of Quantification in Ordinary English, in Hintikka, J. et al (eds), Approaches to Natural Language, 221 - 242, Reidel, Dordrecht.

Moxey, L and A Sanford, 1993, Communicating Quantities, Lawrence Erlbaum Associates, Hove (UK).

Moxey, L and A Sanford, 1996, Choosing the Right Quantifier: Usage in the Context of Communication, in T. Givon (ed), Conversation: Cultural, Cognitive and Communicative Perspectives, 207 - 231, Amsterdam: J Benjamins.

Moxey, L, A Sanford and S Barton, 1990, The control of attentional focus by quantifiers, in K. J. Gilhooly et al (eds), Lines of Thinking: Reflections on the Psychology of Thought,Wiley, 1990.

Neale, S, 1990, Descriptions, Bradford Books, MIT Press, Cambridge, Mass.

Percus, O, T Gibson and S Tunstall, 1997, Antecedenthood and the evaluation of quantifiers, Unpublished paper presented at the CUNY Processing Conference.

Sanford, A, L Moxey and K Patterson, 1996, Attentional focusing with quantifiers in production and comprehension, in Memory and Cognition 24 (2), pp 144155.

Zwarts, F, 1996, Facets of Negation, in J. van der Does, and J van Eijck, (eds), Quantifiers, Logic and Language, pp 385 - 421, CSLI, Stanford. 